\title{
Research on Numerical Methods of Differential /Algebraic Equations for Multibody System Dynamics
}

\author{
Fangfang Fu \\ Nanchang Institute of Science \& Technology, Nanchang City, Jiangxi \\ Province, China, 330108
}

\begin{abstract}
The dynamic model of multibody system is one of the main contents of dynamic study of multibody system. The dynamic mathematical model can be divided into two categories, the first one is a simple second order differential equation; the second one is Differential - Algebraic Equations. As for the solution of the first kind of equation has matured, but the solution of the second class of equations is still in the exploratory stage, not perfect, is a difficult problem.

Keywords numerical methods, differential algebraic equations, multibody system dynamics

\section{Introduction}

Multi-body system is defined by a number of rigid body or rigid body and elastomer through a certain way to connect each other to form a complex mechanical system. The dynamics of multi-rigid-body system is based on the fact that each component in the system is abstracted as rigid body, but it can take into account the influence of elasticity and damping at the joint point of each component. Multi-body system dynamics has been developed on the basis of classical mechanics. From 1960s to now, multi-body system dynamics modeling has formed many unique methods, such as Newton-Euler method, Lagrange method, Roberson-Wittenburg Method, Kane method, Huston method and so on, and has achieved quite perfect results, has solved the engineering many practical application issues.
\end{abstract}




\section{The numerical method for multibody system dynamic equation}

Due to its strong coupling and high nonlinearity, the dynamic model of flexible multibody system can not get the analytic solution of the response, and it needs to rely on the numerical simulation to realize the dynamic analysis of the system. The numerical method is directly related to the dynamic model related. The commonly used Lagrange method is one of the general methods to establish the dynamic equations of multibody systems. With the deepening of the research contents of multibody system dynamics and the expanding of the research range, the traditional Lagrange equations can be divided into the first and second The former is used for the generalized coordinates independent of the particle system, the kinetic equation for the ordinary differential equations; the latter applies to the generalized coordinates are not completely independent of the particle system, the dynamic equation for differential-algebraic equations. For the multibody system with complete and constant constrains, when the generalized coordinate takes the hinge coordinate and the modal coordinate of the deformable body, the generalized coordinates of the system are independent, and the first kind of Lagrange equation is used. The dynamic equation For the following forms:

$$
M(q, t) \ddot{q}+M(q, t) \dot{q}-\frac{\partial T}{\partial q}=Q
$$

The expression is a set of second-order ordinary differential equations, the unknown is the generalized coordinates, the number of equations equal to the number of equations, are equal to the number of generalized degrees of freedom. This is the smallest number of closed differential equations, the solution method can be directly followed the first-order ordinary differential equation numerical method. For example: Runge-kutta method, Adams-Bashforth show multi-step method and so on.

In this paper, we mainly study the Lagrange equation of the second kind: we introduce the Lagrangian multiplier $\lambda$ and increase the constraint equation $C(q, t)$ $=0$, the expression is as follows:

$$
\begin{gathered}
M(q, t) \ddot{q}+k q+C_{q}^{T}(q, t) \lambda=Q_{f}+Q_{V} \\
C(q, t)=0
\end{gathered}
$$

$(\mathrm{Q}, \mathrm{t}) \in \mathrm{Rnq} \in \mathrm{R} \lambda \in \mathrm{mR}$ is the Lagrange multiplier vector, $\mathrm{C}(\mathrm{q}, \mathrm{t}) \in \mathrm{mR}$ is the left part of the system constraint equation, and $C(q(t))$ is the left-hand side of the system constraint equation. $(\mathrm{T})=\mathrm{q} \partial \mathrm{C}(\mathrm{q}, \mathrm{t}) / \partial \mathrm{q}$ is a Jacobi matrix, $\mathrm{knxn} \in \mathrm{R}$ is a stiffness matrix, which is the generalized dominant force and is the generalized force related to the quadratic velocity.

This kind of characteristic equation is called DAE (Differential Algebraic Equations) equation, this is the concrete research object of the numerical method of this text. In the following chapters, we will introduce the numerical methods for solving this kind of equations. We introduce the Moore-Penrose generalization in the numerical solution before the introduction of these methods. 
The basic theory of inverse and the direct numerical integration method of ordinary differential equations, which lays the foundation for the discussion of the solution of differential-algebraic equations.

\section{Generalized Inverse Solution of M - P}

In the process of solving differential-algebraic equations, the Jacobian matrix of the constraint equation is often a singular matrix or an arbitrary rectangular matrix, which does not have the usual inverse matrix, which brings difficulties to the solution. In 1920, EHMoore first introduced the concept of generalized inverse matrices, which did not attract attention for the next thirty years. Until 1955, R. Penrose gave a more definite form of Moore's generalized inverse matrix , The study of generalized inverse matrices has entered a new period and has been applied in many fields such as mathematical statistics, system theory, optimization theory, modern control theory and so on.

The two commonly used algorithms for solving generalized inverse are:

Gaussian elimination method is actually a matrix decomposition method for solving linear equations of a common method, the method can also be used for the matrix LUA full rank decomposition. The matrix $A$ is denoted by its $m \times n$ elements (), and assuming that rank $(\mathrm{A})=\mathrm{r}$, the matrix $\mathrm{A}$ is selected by multiple selections and eliminated:

Where $\mathrm{L}$ is a unit trapezoidal matrix of order $\mathrm{m} \times \mathrm{r}$, and $\mathrm{U}$ is an upper trapezoidal matrix of order $\mathrm{r} \times \mathrm{n}$. The generalized inverse of $\mathrm{A}$ is:

$$
A=U^{T}\left(U U^{T}\right)^{-1}\left(L^{T} L\right)^{-1} L^{T}
$$

Gaussian elimination method is relatively simple, the calculation is small, high efficiency, but easily lead to rank loss, so when the structure is more stable or clear from the physical sense of the rank of the matrix, this method can be used.

\section{The numerical integral method for differential equations of multibody system dynamics}

In recent twenty years, many numerical methods for solving multibody dynamical equations have been developed. Many commonly used direct numerical methods with first-order differential equations, such as the first-order differential equations, Integral method, direct numerical integration method of second-order differential equation and so on.

In many practical physical or chemical processes that can be described by ordinary differential equations, there are often many complex subprocesses and interactions between them, some of which exhibit rapid changes, and others that are relatively Slow changes, and the rate of change can vary by a very large magnitude, and the solutions to the ordinary differential equations describing these processes will also contain fast and slow components. If the difference between the fast-changing and slow-changing processes in a process is very large, 
it is mathematically called a rigid process, and the ordinary differential equation describing the process is called the rigid equation, Ill-conditioned equation or bad condition equation. From a numerical point of view, in order to obtain the fast component of the response, this time should be integrated with small time steps since it is often unrealistic to use such a small step size for integration over a fairly long integration interval On the one hand, when the fast component has stabilized or disappeared, it should use a larger time step integration. But the theory and practice shows that many methods, especially the explicit method of step size can not be enlarged, otherwise there will be numerical instability and the phenomenon of rapid increase in error, so that cover up the true solution. It is precisely because of this nature, making the traditional ordinary differential equations numerical integration has encountered great difficulties.

The solution of differential-algebraic equations of flexible multibody systems can be reduced to solving a set of second-order ordinary differential equations. Usually there are two ways to solve this kind of second-order differential equations. There are many monographs on the stability of this numerical integration method and its solutions. The main contributions are as follows: Wilson's q $=\mathrm{f}(\mathrm{q}, \mathrm{q}, \mathrm{t}) \& \& \& \theta$ method, Parker's Stiffness Method, Newmark? Method.

The methods of converting differential-algebraic equations into numerical models are AugmentedMethod and Condyne Eliminitaed Method. The augmented method is a Lagrangian multiplier as a unified treatment of unknowns, the larger the number of variables to be closed equations. This method usually uses the direct integration method, because the solution process is at the constraint level of acceleration, and the numerical solution may diverge due to rounding error. The Baumgarte constraint violation stability method well overcomes the shortcomings of the direct integration method. As for the contraction method, it essentially establishes the minimum number of equations numerically. In the implementation process, it is embodied in the treatment of constraint equations. The abbreviation method includes null space method, LU decomposition method and QR decomposition method. (3) N-R minimal norm solution correction method; (4) constraint error self-stabilization method and other solutions of differential-algebraic equations. (4) Constrained error selfstabilization method and other solutions of differential-algebraic equations.

In the dynamic analysis of multi-body system, the direct integration method (numerical integration method) the basic idea: not to make any transformation equation directly using step-by-step numerical integration solution. This solution solves the kinetic equations separately in discrete steps with interval steps, and must be known for solutions at discrete points from 0 to $\mathrm{t}$ at the time $\Delta \mathrm{tt}+\Delta \mathrm{t}$. It is clear that the number of operations required for the numerical integration method is proportional to the number of time steps in the analysis. In general, the direct integration method is very effective in calculating the short-term dynamic response. The direct integration method is based on the following two points: (1) the differential equations of motion are satisfied only at discrete discrete intervals of time and not at any one time; and (2) the variations of the displacements, velocities and accelerations at each time $\Delta \mathrm{t} \Delta \mathrm{t}$ are assumed. 
The basic idea of NR iterative minimum norm solution is that: On the basis of the constraint - restrained stability method, the basic theory of $\mathrm{M}$ - P generalized inverse matrix and NR iterative method are used to solve the constraint equation. Amendment. First, the numerical solution of the generalized coordinates at the time of it $=t$ is obtained by the constraint stability method, in which the default of the displacement constraint equation occurs when the default of the displacement constraint equation exceeds the given precision range.

In the numerical computation, it is sometimes necessary to decompose the generalized coordinates independently. On the one hand, for multi-rigid-body systems, the generalized coordinate independence decomposition of the system can be carried out automatically by the computer. However, for flexible multibody systems with strong nonlinearities and high degrees of freedom, the decomposition of independent coordinates automatically by computer can cause instability of numerical solutions. This is mainly due to the elastic deformation of the components of the system. The inverse kinematics of deformation is uncertain. On the other hand, from a numerical point of view, in order to obtain the fast component of the response, this time should be integrated with a small time step since the integration process with such a small step length can be understood in a considerably long integration interval, And secondly, when the fast component has stabilized or disappeared, it should be integrated with a larger time step. However, there are contradictions between theory and practice. For example, there are many numerical algorithms, especially the explicit step method. Otherwise, numerical instabilities and error divergence will occur. It is precisely because of this characteristic that the numerical integration of differential equations has encountered great difficulties, so in dealing with this problem, try to use the correction method, when the time will increase the numerical stability, but at the same time a problem is Control of Default Value. In addition, the Jacobian constraint matrix of the constraint equation is decomposed according to the independent generalized coordinates, and the relationship between the correlation coordinates and the independent coordinates can be done by numerical algorithm. To complete the numerical algorithm need to ensure that the constraint matrix coefficient matrix full rank decomposition, generally can be used QR orthogonal method, LU method. In the choice of independent coordinates, because the object contains flexible body, the need for human intervention. If the flexible coordinate is chosen as the relevant coordinate of the system, numerical instability is easily caused by the Newton-Raphson method. Therefore, the decomposition of generalized coordinates should be worthy of attention.

\section{Conclusion}

The research on numerical algorithm of multibody system dynamics has great application value and theory meaning to the development of aerospace, machinery and high-speed structure. In the development of multibody dynamics, the deformation motion of flexible bodies and the large-scale spatial motion of 
flexible bodies occur at the same time and their mutual coupling bring a lot of difficulties to the numerical calculation of dynamics.

\section{References}

[1] Lin Weixin, Liu Mingzhi, YANG Qing-zhi.Numerical Solution of Dynamic Equation of a New Flexible System. Mechanical Product Development \& Innovation 55(12), pp.68-70, 2011

[2] Wang Guoping .Numerical solution of multi-body system dynamics. Computer Simulation. 8 (5), pp.87- 91, 2013

[3] Wu Guorong, Chen Fuquan, Tang Ruilin.A New Method For Solving The Dynamic Equations Of Flexible Multibody Systems. Acta Mechanica Sinica. 12(10), pp. 18- 20, 2012

[4] YANG Hui, HONG Jia-zhen, YU Zheng-yue.Dynamic modeling and numerical simulation of rigid-flexible coupled multi-body system. Chinese Journal of Computational Mechanics, 9(6), pp.58- 61, 2011

[5] Wang Baoyuan.Multi-body system dynamics software technology. Journal of Computer Applications and Software, 8(4), pp.85-87, 2014 